\title{
Ageing analysis of the giant radio galaxy $\mathrm{J} 1343+3758$
}

\author{
M. Jamrozy ${ }^{1}$, J. Machalski ${ }^{2}$, K.-H. Mack ${ }^{3,1}$, and U. Klein ${ }^{1}$ \\ 1 Radioastronomisches Institut der Universität Bonn, Auf dem Hügel 71, 53121 Bonn, Germany \\ e-mail: [mjamrozy;uklein@astro.uni-bonn.de] \\ 2 Obserwatorium Astronomiczne, Uniwersytet Jagielloński, ul. Orla 171, 30244 Kraków, Poland \\ e-mail: machalsk@oa.uj.edu.pl \\ 3 Istituto di Radioastronomia, via P. Gobetti 101, 40129 Bologna, Italy \\ e-mail: mack@ira.cnr.it
}

Received 14 September 2004 / Accepted 22 November 2004

\begin{abstract}
Deep 4860 and $8350 \mathrm{MHz}$ observations with the VLA and 100-m Effelsberg telescopes, supplementing available radio survey maps at the frequencies of $327 \mathrm{MHz}$ (WENSS survey) and $1400 \mathrm{MHz}$ (NVSS survey), are used to study the synchrotron spectra and radiative ages of relativistic particles in opposite lobes of the giant radio galaxy J1343+3758 (Machalski \& Jamrozy 2000). The classical spectral ageing analysis (e.g. Myers \& Spangler 1985) with assumption of equipartition magnetic fields gives a mean separation velocity $\left(\left\langle v_{\mathrm{sep}}\right\rangle\right)$ of about $0.16 c$ and $0.12 c$ measured with respect to the emitting plasma, and suggests a maximum particle age of about 48 and $50 \mathrm{Myr}$ in the NE and SW lobes, respectively. On the contrary, a mean jethead advance speed $\left(\left\langle v_{\text {adv }}\right\rangle\right)$ in the above lobes, derived from ram-pressure arguments, is about $0.016 c$ and $0.017 c$, respectively. This would imply a substantial backflow of the lobe material from the hotspot regions towards the radio core, $v_{\mathrm{bf}}$, which is not supported by the available radio maps. A compromise is achieved by assuming an enhancement of 3 to 5 times the equipartition magnetic field strengths in the lobes which gives $\left\langle v_{\text {sep }}\right\rangle \approx 0.06 c$ and $\left\langle v_{\text {adv }}\right\rangle \approx\left\langle v_{\text {bf }}\right\rangle \approx 0.03 c$, hence a dynamical age of the source of $204 \pm 40 \mathrm{Myr}$. A comparison of the radiative and dynamical ages of the investigated giant radio galaxy implies that the dynamical age is about 4 times the maximum synchrotron age of the emitting particles, which supports the conclusion of Blundell \& Rawlings (2000) that the spectral and dynamical ages are comparable only when they are $\ll 10 \mathrm{Myr}$, and suggests that for FRII-type sources the discrepancy between these ages increases with age.
\end{abstract}

Key words. radio continuum: galaxies - galaxies: active - galaxies: individual: J1343+3758

\section{Introduction}

Reliable determinations of the present (at the observing epoch) age of extended extragalactic radio sources are crucial for an observational constraint of the existing analytical models of their dynamical evolution (e.g. Kaiser et al. 1997 (hereafter referred to as KDA); Blundell et al. 1999a). Most of the published studies about the age of the sample members in selected sets of radio sources have been based on the analysis of their radio spectra. There is no doubt that the radio continuum spectra in different parts of a radio source should contain crucial information about its history. In the classical synchrotron-source theory the observed variations of these spectra are directly related to the mechanisms of particle energy losses. If high energy particles with an initial power-law spectrum experienced only synchrotron losses via the uniform magnetic field in which they were immersed, then a characteristic frequency break in that spectrum $v_{\mathrm{b}} \propto B^{-3} t^{-2}$ would appear, where $t$ is the time elapsed since the particles were accelerated to their initial energy distribution (cf. Kardashev 1962; Pacholczyk 1970; Jaffe \& Perola 1973).

This simple method was used to estimate the radiative ages and expansion speeds in large samples of powerful
3CR sources (Myers \& Spangler 1985; Alexander \& Leahy 1987; Leahy et al. 1989; Carilli et al. 1991; Liu et al. 1992), as well as in samples of low- and intermediate-luminosity radio galaxies (Klein et al. 1995; Parma et al. 1999). In the meantime serious criticism of the interpretation of the radio spectrum variations within a source in terms of particle ageing has been published in a number of papers (e.g. Rudnick et al. 1994; Jones et al. 1999; Blundell \& Rawlings 2000) envisaging a possibly dominant rôle of the local magnetic field evolution. Nevertheless, Kaiser (2000) has argued that if the bulk backflow and energy losses, both radiative and adiabatic, "are selfconsistently taken into account", the spectral and dynamical ages (the observed linear size of a source/lobe divided by the speed at which it has expanded to that time) can be comparable. Blundell \& Rawlings (2000) contend that this will be the case only if these ages are $\ll 10 \mathrm{Myr}$.

The determination of the ages of the largest FRII-type (Fanaroff \& Riley 1974) sources is of special interest. Classical double radio sources with a projected linear size $D$ exceeding $1 \mathrm{Mpc}$ (for $\Omega \equiv \Omega_{\mathrm{M}}+\Omega_{\Lambda}=1$ and $H_{0}=50 \mathrm{~km} \mathrm{~s}^{-1} \mathrm{Mpc}^{-1}$ ) 1 are commonly considered as a somewhat special class of

\footnotetext{
${ }^{1}$ Scaling $H_{0}$ to a more actual value does not scale the age.
} 
extragalactic sources. They are characterised by the rapid decrease of their luminosity with increasing size, which is clearly visible in the low-frequency luminosity vs. projected linear size (the $P-D$ ) diagram, cf. Neeser et al. (1995); Blundell et al. (1999a). The recent analytical models of the time evolution of FRII-type sources (KDA; Blundell \& Rawlings 1999b) can explain the extreme source size by the longer lifetimes of the largest sources and effective synchrotron cooling, adiabatic expansion losses, and inverse Compton scattering of the cosmic microwave background. However, giants can be exceptionally large also because their intergalactic environment is less dense than that surrounding smaller sources (e.g. Mack et al. 1998). The latter suggestion has been supported by the finding that high-redshift giants studied by Cotter (1998) exist exclusively in low-density environments.

Our recent analysis of the time evolution of giant radio galaxies in view of the analytical KDA model strongly suggested that there is not one dominant factor responsible for the huge linear sizes of those sources; we found that giants are relatively old sources with high enough jet power that have evolved in a low-density environment (Machalski et al. 2004a).

In this paper we perform the standard ageing analysis for the source J1343+3758, the third largest FRII-type radio galaxy known after 3C 236 and WNB 2147+816 (Machalski \& Jamrozy 2000), following the previous similar studies of giant sources by Parma et al. (1996), Mack et al. (1998), Schoenmakers et al. $(1998,2000)$ and Lara et al. (2000). This ensures a homogeneous comparison of the ages determined for giant radio sources. We also estimate the dynamical age of this source using the approach of Begelman \& Cioffi (1989).

In Sect. 2 the observational data used for the spectral ageing analysis are described, and the non-survey high-frequency 4860 and $8350-\mathrm{MHz}$ maps of $\mathrm{J} 1343+3758$ are presented. Its morphological, spectral and polarisation properties are described in Sect. 3. The spectral ageing analysis and the dynamical considerations are given and the results are discussed in Sect. 4. Other physical parameters of the investigated source (the jet power, central core density, pressure of the shocked jet material, total energy contained in the lobes, and fraction of the jet energy used for the adiabatic expansion of the source cocoon) are calculated and discussed in Sect. 5. The summary is given in Sect. 6.

\section{Observational data}

Below we describe the observational data used for the spectral ageing analysis of the source $\mathrm{J} 1343+3758$. For this purpose we incorporate the low-resolution 327 and $1400-\mathrm{MHz}$ maps available from the WENSS (Rengelink et al. 1997) and NVSS (Condon et al. 1998) sky surveys, respectively, supplemented by our own high-frequency 4860 and $8350-\mathrm{MHz}$ maps. In these maps J1343+3758 appears as a straightforward 11!3 FRII-type radio source composed of two separate lobes without any evident bridge connecting them.

\subsection{WENSS and NVSS maps}

The WENSS map of the total intensity emission at $327 \mathrm{MHz}$ with an angular resolution of $54^{\prime \prime} \times 87^{\prime \prime} \cdot 7$ is reproduced in Fig. 1a. The emission from the south-west (SW) lobe extends almost up to the radio core region, and is described in more detail in Sect. 3.1. The total intensity 1400-MHz NVSS map with a resolution of $45^{\prime \prime} \times 45^{\prime \prime}$ and the electric field $\boldsymbol{E}$-vectors superimposed is shown in Fig. 1b. Figure 1c shows the linearly polarised intensity map with the vectors of fractional linear polarisation superimposed. The mean fractional polarisation in both lobes at $1400 \mathrm{MHz}$ is given in Sect. 3.3. A distinct hotspot at the outer edge of the north-east (NE) lobe is visible on the high-resolution 1400-MHz FIRST (Becker et al. 1995) map. A trace of a hotspot is also visible in the SW lobe although it is rather dim.

\subsection{VLA observations}

The observations were carried out with the VLA D-array at two correlator frequencies of 4835 and $4885 \mathrm{MHz}$, each with a bandwidth of $50 \mathrm{MHz}$. The telescope gains were calibrated using the standard calibration source 3C286 and the flux density scale of Baars et al. (1977). The source J1324+408 was used as phase calibrator. The SW lobe was observed in Sept. 30, 2000 with the pointing center at $\alpha_{\mathrm{J} 2000}=13^{\mathrm{h}} 42^{\mathrm{m}} 39^{\mathrm{s}} ; \delta_{\mathrm{J} 2000}=$ $37^{\circ} 56^{\prime} 00^{\prime \prime}$, i.e. $-3^{\mathrm{m}} .84$ away from the core. With an integration time of $2 \times 31 \mathrm{~min}$, a rms noise of about $27 \mu \mathrm{Jy}^{\text {beam }}{ }^{-1}$ was achieved. The opposite NE lobe was observed in Feb. 25, 2003 with the pointing center at $\alpha_{\mathrm{J} 2000}=13^{\mathrm{h}} 43^{\mathrm{m}} 17^{\mathrm{s}} ; \delta_{\mathrm{J} 2000}=$ $38^{\circ} 01^{\prime} 00^{\prime \prime}$, i.e. $+5^{\mathrm{m}} \cdot 18$ away from the core. With an integration time of $3 \times 35 \mathrm{~min}$, the rms noise was about $23 \mu \mathrm{Jy}$ beam $^{-1}$. The data were reduced and maps of both lobes produced using the NRAO AIPS software package. After initial CLEANing of the maps, several self-calibrations were performed to improve their quality. Then the maps of the NE and SW lobes were corrected for the primary beam attenuation and convolved to the common resolution of $20^{\prime \prime} \times 20^{\prime \prime}$. Finally, using the AIPS task COMB, the two maps were combined into a single image of the entire source. This combined $4860-\mathrm{MHz}$ map overlaid on the optical DSS field is shown in Fig. 2. The contour levels are spaced by a factor of $\sqrt{2}$ starting with $40 \mu \mathrm{Jy}_{\text {beam }}{ }^{-1}$. The VLA data can be affected by missing-spacing effects, which implies that the measured $4860 \mathrm{MHz}$ flux densities in the extended diffuse region of the lobes can be lower limits. The flatspectrum radio core (cf. Table 1$)$ coincides with a $17^{\mathrm{m}} .45(R-$ band) galaxy. Its redshift of $0.2267 \pm 0.0005$ was measured by Machalski et al. (2001).

\subsection{Effelsberg observations}

The second set of high-frequency observations of J1343+3758 has been carried out at $8350 \mathrm{MHz}$ with a broad-band $(1100 \mathrm{MHz})$ receiver in the secondary focus of the Effelsberg $100-\mathrm{m}$ radio telescope. The system is fed by a single horn with two opposite circularly polarised feeds providing an angular resolution of $82^{\prime \prime} \times 82^{\prime \prime}$. The observations were made in two 

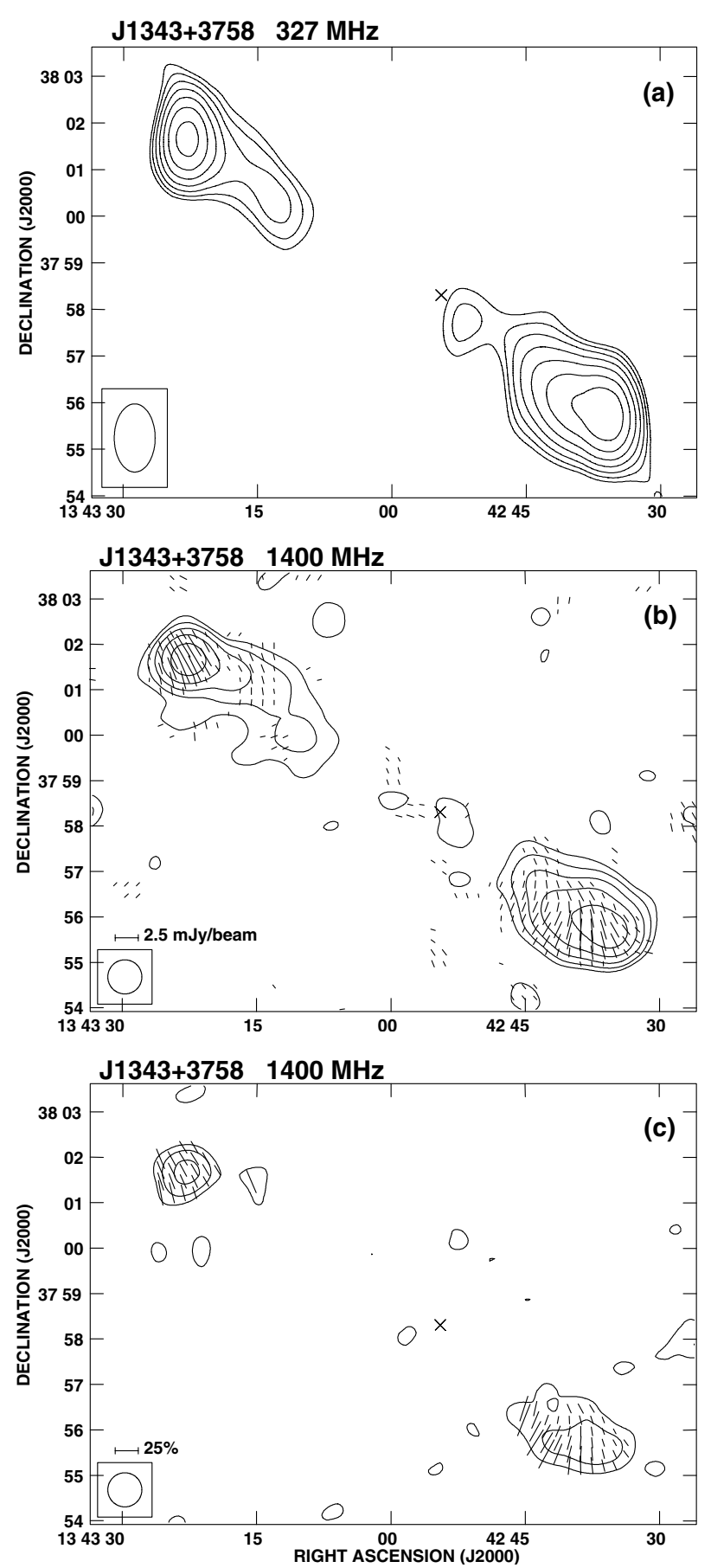

Fig. 1. Images of the radio source J1343+3758 at a) 327 and b), c) $1400 \mathrm{MHz}$ from the WENSS and NVSS surveys, respectively. a) The contours, spaced by factors of $\sqrt{2}$ in brightness, are plotted starting with $9 \mathrm{mJy}_{\text {beam }}{ }^{-1}$. b) Total intensity contours, spaced by a factor of 2 , are plotted starting with $1 \mathrm{mJy}_{\text {beam }}^{-1}$. Superimposed are electric field $\boldsymbol{E}$-vectors with their lengths proportional to the polarised intensity. c) Linearly polarised intensity contours of $0.8,1.6,3.2 \mathrm{mJy}^{\text {beam }}{ }^{-1}$ with the vectors of the fractional linear polarisation superimposed. The scales of the polarisation vectors are indicated by the bars, and the beam size by the ellipse in a) and circles in b), c) in the bottom left corners of the images; "x" marks the position of the parent galaxy.

runs of $6.5 \mathrm{~h}$ each. The weather conditions were excellent during the measurements on Aug. 5 and 6, 2003.
Pointing and focusing of the antenna, as well as the calibration and polarisation performance of the receiver were checked by frequent (every two hours) cross-scanning and mapping of the calibration source 3C 286. Its assumed flux density of $5.225 \mathrm{Jy}$ at $8350 \mathrm{MHz}$ is based on the scale of Baars et al. (1977). The measured mean polarisation position angle and the mean degree of polarised intensity were 34.1 and $11.6 \%$, respectively.

The data reduction was performed using the MPIfR-NOD2 reduction software (Haslam 1974). Individual one-dimensional scans were first transformed into maps of the Stokes $I, Q$, and $U$ signals. Each map was then carefully examined for interference spikes. The scanning effects were removed and a stack of individual maps was made with the technique of "basketweaving" (Sieber et al. 1979). The final total-power and polarised-intensity maps were produced using the NRAO-AIPS package. The applied integration time has allowed us to achieve a rms noise of 0.39 and $0.09 \mathrm{mJy}^{\text {beam }}{ }^{-1}$ in the $I$ map and $Q$, $U$ maps, respectively.

The Effelsberg 8350-MHz map of the total intensity emission from J1343+3758 is shown in Fig. 3a. $\boldsymbol{E}$-vectors of the linearly polarised emission with their lengths proportional to the polarised intensity $\left(1^{\prime \prime}=42 \mu \mathrm{Jy}\right.$ beam $\left.^{-1}\right)$ are superimposed on the total-intensity contours. The contour map of linearly polarised emission with the vectors of the fractional linear polarisation superimposed is shown in Fig. 3b. At this frequency both lobes are highly polarised; the degree of polarisation in the NE and SW lobes is given in Sect. 3.3.

\section{Morphological, spectral, and polarisation properties}

\subsection{Morphology of the source}

The morphology of $\mathrm{J} 1343+3758$ in all of the maps presented above is typical of an FRII-type radio galaxy, although some features are seen only in the WENSS map. As mentioned in Sect. 2.1, at $327 \mathrm{MHz}$ the SW-lobe emission seems to extend back to the compact core region, reaching a separate $15 \mathrm{mJy}$ radio component. As this component coincides with a faint optical galaxy $\left(R \approx 19 \mathrm{~m} 5 ; \alpha_{\mathrm{J} 2000}=13^{\mathrm{h}} 42^{\mathrm{m}} 52^{\mathrm{s}} .47 ; \delta_{\mathrm{J} 2000}=\right.$ $37^{\circ} 57^{\prime} 28^{\prime \prime} .4$ ) and is $55^{\prime \prime} .8$ apart from the radio core and the host galaxy, it is very likely a steep-spectrum background source.

The NE lobe consists of two parts: the brighter northernmost region seen in all four maps and containing the distinct hotspot, and the low-brightness elongated emission aligned with the source axis and extending half way towards the core. The latter part is not detected at $8350 \mathrm{MHz}$. The integrated flux density of the SW lobe is larger than that of the NE one, so the brighter lobe is closer to the host galaxy. Such a property has been found in most of the giant sources in the sample of Machalski et al. (2001). This is expected in the frame of the pure kinematic model of Longair \& Riley (1979): if a source is intrinsically highly symmetric, its inclination to the observer's line of sight differs (but slightly) from $90^{\circ}$, and the luminosity of its lobes decreases with time. In such a case, the lobe seen as closer to the radio core ought to be younger and brighter than the farther one. However, in Sects. 4.2 and 4.3 we find 


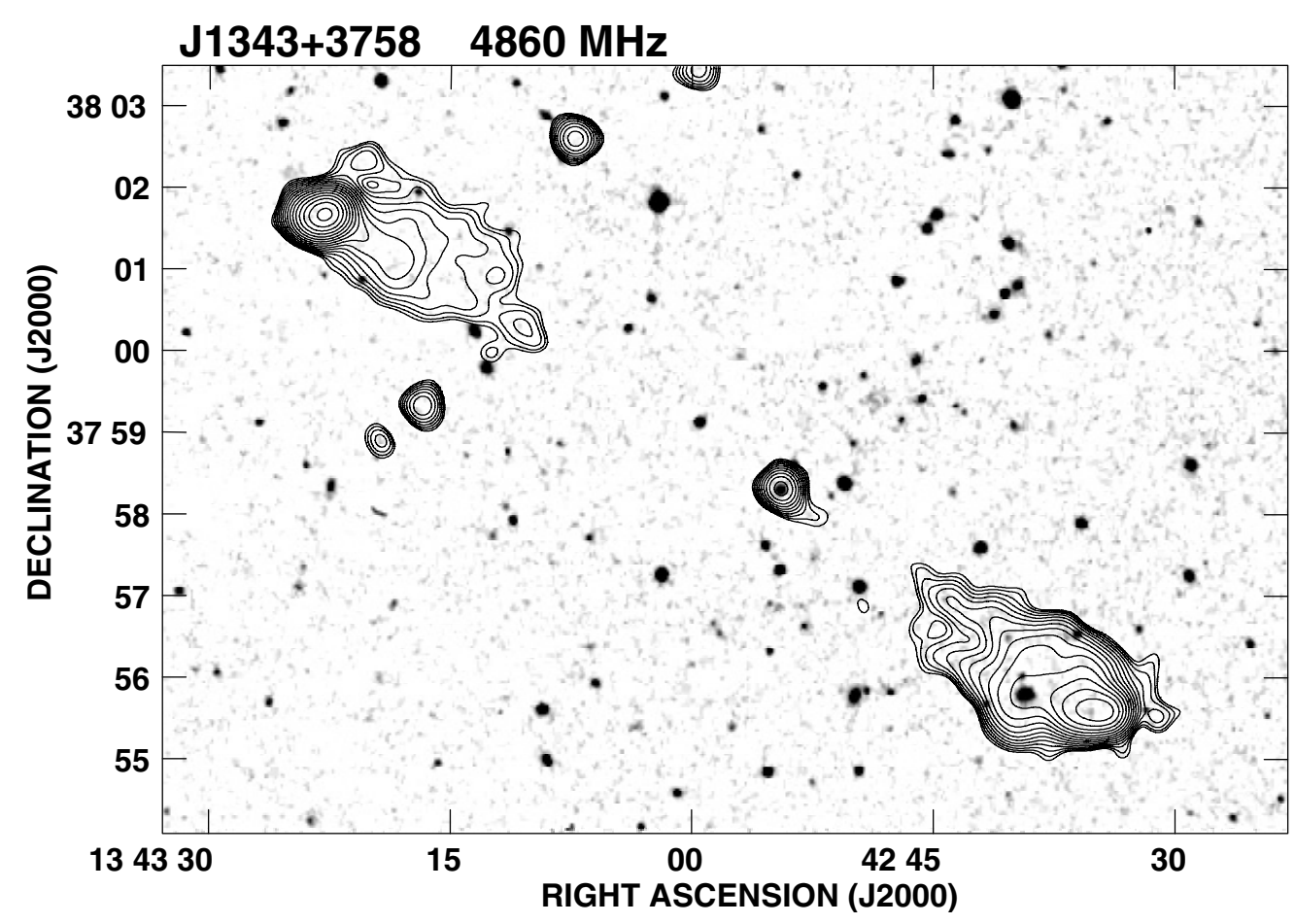

Fig. 2. VLA D-array map of J1343+3758 at $4860 \mathrm{MHz}$ convolved to the angular resolution of $20^{\prime \prime} \times 20^{\prime \prime}$ and overlaid on the optical field from the Digital Sky Survey. The contour levels are spaced by factors of $\sqrt{2}$; the first contour is $40 \mu \mathrm{Jy}_{\text {beam }}{ }^{-1}$. The compact $1.16 \mathrm{mJy}$ radio core coincides with the parent galaxy position.

Table 1. The radio flux densities of the source $\mathrm{J} 1343+3758$ and its lobes.

\begin{tabular}{rlcccc}
\hline \hline Freq. & \multicolumn{5}{c}{ Flux density } \\
& $\begin{array}{c}\text { Total } \\
\text { SW lobe } \\
{[\mathrm{MHz}]}\end{array}$ & $\begin{array}{c}\text { NE lobe } \\
{[\mathrm{mJy}]}\end{array}$ & $\begin{array}{c}\text { Core } \\
{[\mathrm{mJy}]}\end{array}$ & $\begin{array}{c}\text { Ref. } \\
{[\mathrm{mJy}]}\end{array}$ & \\
\hline 151 & $812 \pm 67$ & $564 \pm 57$ & $247 \pm 36$ & & $(1)$ \\
151 & $630 \pm 80$ & $400 \pm 64$ & $230 \pm 50$ & & $(2)$ \\
232 & $620 \pm 130$ & $330 \pm 100$ & $290 \pm 90$ & & $(3)$ \\
327 & $390 \pm 25$ & $243 \pm 20$ & $146 \pm 12$ & & $(4)$ \\
1400 & $140 \pm 7$ & $83 \pm 4$ & $57 \pm 5$ & & $(5)$ \\
1400 & & & & $1.54 \pm 0.15$ & $(6)$ \\
4850 & & $20 \pm 4$ & & & $(7)$ \\
4860 & $36.5 \pm 1.9$ & $19.3 \pm 1.5$ & $16 \pm 1.0$ & $1.16 \pm 0.03$ & $(8)$ \\
8350 & $19.0 \pm 2.3$ & $10.7 \pm 1.8$ & $8.3 \pm 1.5$ & & $(8)$ \\
\hline
\end{tabular}

References: Surveys: (1) 7C (Pooley et al. 1998); (2) 6C (Hales et al. 1988); (3) Miyun (Zhang et al. 1997); (4) WENSS (Rengelink et al. 1997); (5) NVSS (Condon et al. 1998); (6) FIRST (Becker et al. 1995); (7) GB6 (Gregory et al. 1996); (8) this paper.

that the radiative and dynamical ages of the two lobes are very similar, so that the projection effects are likely negligible, and the observed asymmetries must be caused mostly by an inhomogeneity of the intergalactic medium (IGM). The measured flux densities of $\mathrm{J} 1343+3758$ and its components are given in Table 1.

An important parameter of the radio morphology is the "axial ratio" $(A R)$, i.e. the ratio between the length and the width of the source or its lobes (cf. Leahy \& Williams 1984). A low $A R$ suggests a faster lateral expansion of the synchrotron emitting blob of plasma; the expansion is likely dependent on the ratio between the internal pressure in the blob and the pressure of the ambient medium (e.g. Begelman \& Cioffi 1989; KDA). Using the prescription given by Leahy \& Williams (1984), values of $A R$ of $4.8 \pm 0.9$ and $2.9 \pm 0.6$ are found for the $\mathrm{NE}$ and SW lobes of $\mathrm{J} 1343+3758$. These values are similar to those determined in another sample of giant radio sources by Schoenmakers et al. (2000) where most of the lobes have $2 \lesssim A R \lesssim 5$.

\subsection{Spectral index map}

We have made spectral index maps of $\mathrm{J} 1343+3758$ using the WENSS, NVSS, VLA, and Effelsberg data. In each case the highest resolution map was convolved to the resolution of another map. The final gray-scale map of the spectral index between the frequencies 327 and $4860 \mathrm{MHz}$ is shown in Fig. 4a. The rapid steepening of this index from the hotspot regions towards the radio core is evident.

The high-resolution FIRST and our VLA maps (cf. Machalski \& Jamrozy 2000) allow us to determine the size and spectral index of the compact hotspot in the NE lobe. Its Gaussian deconvolved size is $\left(5^{\prime \prime} .8 \pm 0.0^{\prime} 4\right) \times\left(22^{\prime \prime} 7 \pm 00^{\prime} .1\right)$ with the position angle tilted by $\sim 20^{\circ}$ from the source main axis. The spectral index is $\alpha_{4860}^{1400}=0.68 \pm 0.05\left(S_{v} \propto v^{-\alpha}\right)$. The hotspot linear size is used in the dynamical considerations in Sect. 4.3.

\subsection{Polarisation properties}

The magnetic field vectors found in this giant source follow the general trend known for FRII-type radio galaxies (Saikia $\&$ Salter 1988). The magnetic field lines are circumferential 

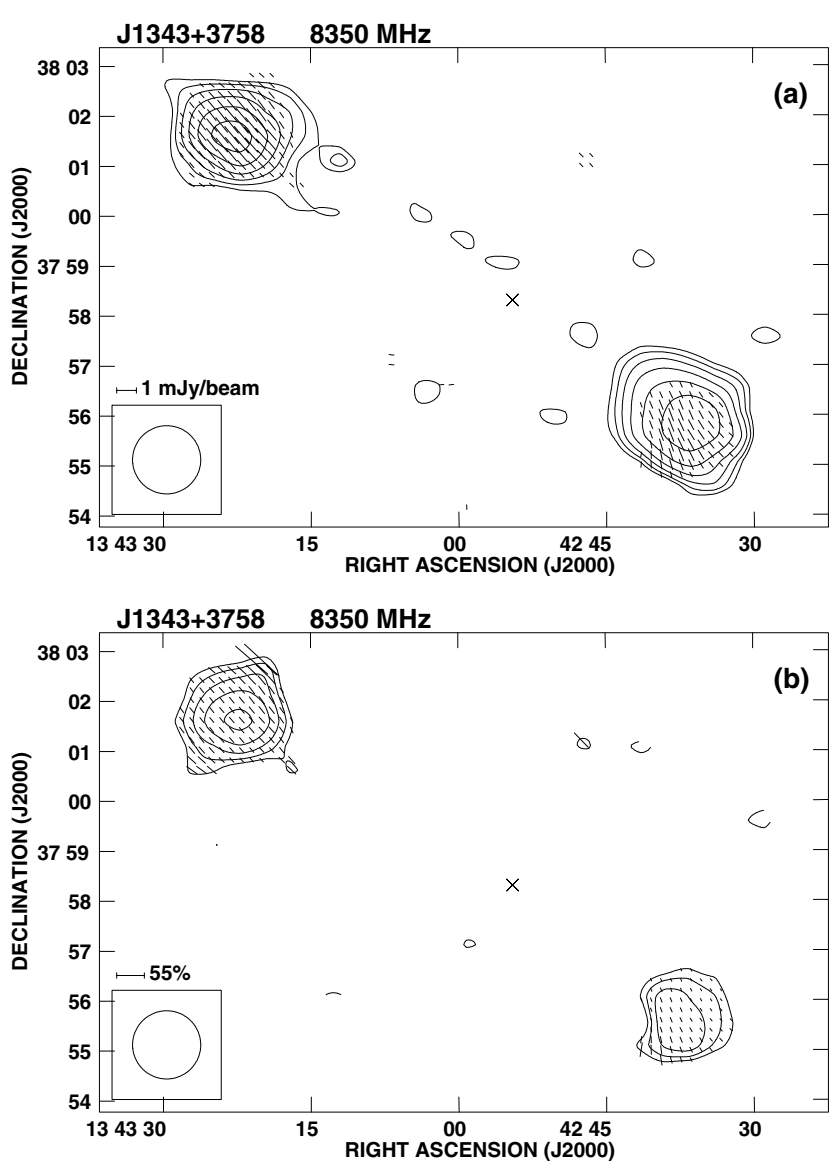

Fig. 3. The Effelsberg images of the source J1343+3758 at $8350 \mathrm{MHz}$. a) Total intensity contours spaced by a factor of $\sqrt{2}$ starting with $1.35 \mathrm{mJy}_{\text {beam }}{ }^{-1}$. Superimposed are $\boldsymbol{E}$-vectors with their lengths proportional to the polarised intensity. b) Linearly polarised intensity contours spaced by a factor of $\sqrt{2}$ starting with $0.28 \mathrm{mJy} \mathrm{beam}^{-1}$ with the vectors of the fractional linear polarisation superimposed. Otherwise the layout is the same as in Fig. 1.

towards the sources' peripheries and nearly orthogonal to the source axis in the vicinity of the hotspots.

The lobes of $\mathrm{J} 1343+3758$ show high fractional polarisation which increases significantly in the neighbourhood of the hotspots (see Figs. 1b and 1c, and Fig. 3). Integration of the available Stokes $Q$ and $U$ images yields a total fractional polarisation of $11.4 \% \pm 0.5 \%$ and $12.3 \% \pm 1.0 \%$ for the NE lobe, and $10.2 \% \pm 0.4 \%$ and $5.3 \% \pm 0.7 \%$ for the SW lobe at 1400 and $8350 \mathrm{MHz}$, respectively. The degrees of polarisation in the SW lobe indicate some depolarisation at higher frequencies.

In order to obtain a Rotation Measure $(R M)$ image of $\mathrm{J} 1343+3758$ we convolved the $1400 \mathrm{MHz}$ polarisation map to the beam of the Effelsberg 8350-MHz image. Using the AIPS task COMB we calculated the values of the $R M$ in the regions where the polarised intensity is larger than $\sim 3 \times$ rms noise at both frequencies. For the Faraday rotation in a slab with a uniform field one can write $R M=(\Delta \phi+\pi n) /\left(\lambda_{1}^{2}-\lambda_{2}^{2}\right)$ where $\Delta \phi \equiv \phi_{1}-\phi_{2}$ is the rotation of the polarisation position angle $\phi$ between the two wavelengths $\lambda_{1}$ and $\lambda_{2}$, where $\lambda_{2}<\lambda_{1}$ and $n$ is an integer. We find a mean $R M$ of $-5.7 \pm 0.9 \mathrm{rad} \mathrm{m}^{-2}$ and $-6.0 \pm 2.1 \mathrm{rad} \mathrm{m}^{-2}$ for the NE and SW lobes, respectively. The $R M$, however, cannot be determined reliably from our
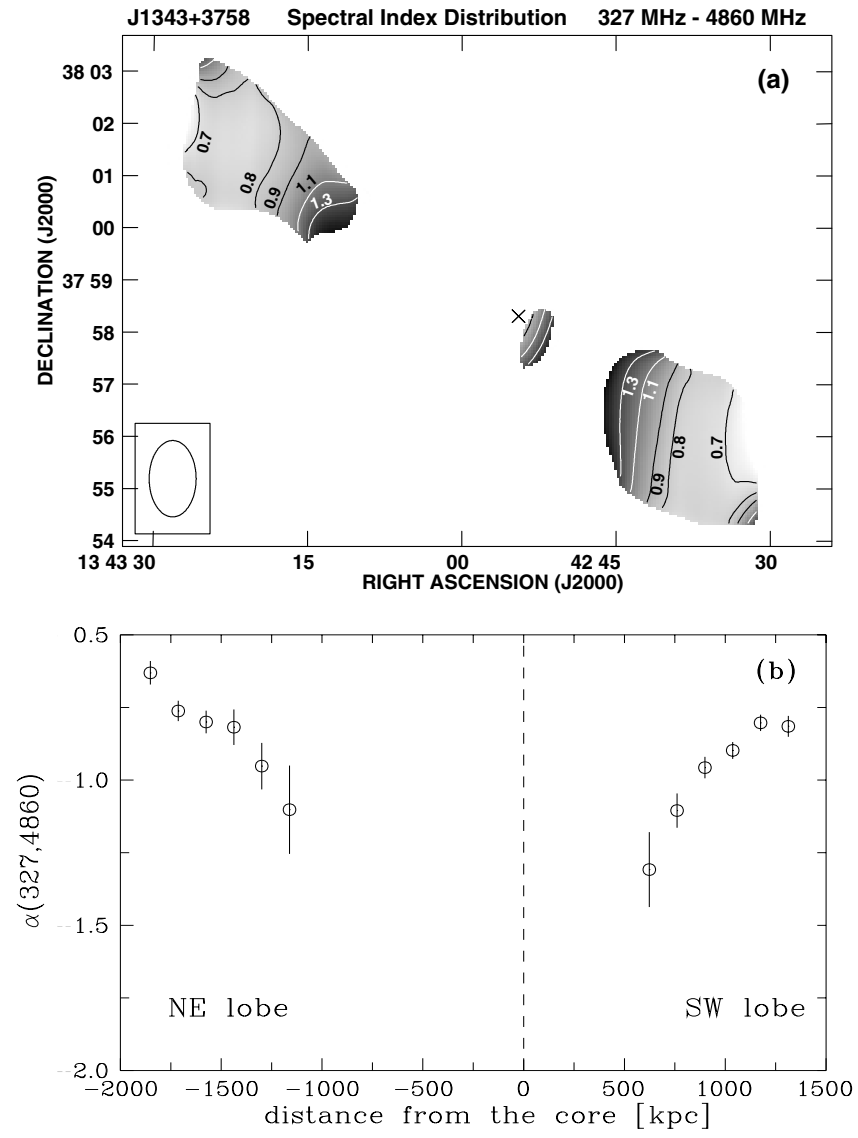

Fig. 4. a) The map of the spectral index $\alpha_{4860}^{327}$ across the radio galaxy; b) this index in $30^{\prime \prime}$ wide strips perpendicular to the source axis vs. strip distance from the core.

two-frequency data because of an ambiguity in the value of $n$. The similar distributions of the magnetic field position angles and of the $R M$ values across the lobes would suggest that a contribution of the radio galaxy to the observed $R M$ is negligible and that observed Faraday rotation may be of Galactic origin since the mean Galactic $R M$ at the position of J1343+3758 lies between $-30 \mathrm{rad} \mathrm{m}^{-2}$ and $0 \mathrm{rad} \mathrm{m}^{-2}$ (Simard-Normandin \& Kronberg 1980). However, this has to be checked by additional polarisation measurements at other frequencies.

\section{Spectral ageing analysis}

\subsection{The method}

For the spectral analysis, the maps obtained at the four different frequencies were convolved to a common angular resolution determined by the lowest original one, i.e. the resolution of the Effelsberg map. Then we determined the spectrum at different positions of the source averaging the four flux densities within $30^{\prime \prime}$-wide strips orthogonal to the source axis. In Fig. $4 \mathrm{~b}$ the spectral index within these strips, calculated between the frequencies of 327 and $4860 \mathrm{MHz}$ is plotted against the physical distance from the radio core.

For the three $60^{\prime \prime}$-wide strips covering the NE lobe and the other three covering the SW lobe, the values of the equipartition energy density $\left(u_{\mathrm{eq}}\right)$ and the corresponding magnetic field 
Table 2. Magnetic field strength, equipartition energy density, and corresponding minimum pressure in the lobes of $\mathrm{J} 1343+3758$ at the given distance from the core.

\begin{tabular}{lrcc}
\hline \hline $\begin{array}{l}\text { Distance } \\
{[\mathrm{kpc}]}\end{array}$ & $\begin{array}{c}B_{\text {eq }} \\
{\left[\eta^{-2 / 7} \mathrm{nT}\right]}\end{array}$ & $\begin{array}{c}u_{\mathrm{eq}} \\
{\left[\eta^{-4 / 7} \mathrm{~J} \mathrm{~m}^{-3}\right]}\end{array}$ & $\begin{array}{c}p_{\min } \\
{\left[\eta^{-4 / 7} \mathrm{~N} \mathrm{~m}^{-2}\right]}\end{array}$ \\
\hline NE lobe & & & \\
1713 & $0.73 \pm 0.15$ & $(4.9 \pm 2.1) \times 10^{-13}$ & $(1.6 \pm 0.7) \times 10^{-13}$ \\
1437 & $0.11 \pm 0.01$ & $(1.1 \pm 0.2) \times 10^{-14}$ & $(3.8 \pm 0.1) \times 10^{-15}$ \\
1161 & $0.084 \pm 0.01$ & $(6.5 \pm 1.2) \times 10^{-15}$ & $(2.2 \pm 0.4) \times 10^{-15}$ \\
SW lobe & & & \\
623 & $0.10 \pm 0.01$ & $(9.3 \pm 2.0) \times 10^{-15}$ & $(3.1 \pm 0.7) \times 10^{-15}$ \\
899 & $0.13 \pm 0.01$ & $(1.5 \pm 0.3) \times 10^{-14}$ & $(4.9 \pm 0.8) \times 10^{-15}$ \\
1175 & $0.55 \pm 0.07$ & $(2.8 \pm 0.7) \times 10^{-13}$ & $(1.9 \pm 0.2) \times 10^{-13}$ \\
\hline
\end{tabular}

strength $\left(B_{\text {eq }}\right)$, calculated with the formula of Miley (1980), as well as the minimum pressure $p_{\min }=\left(\Gamma_{\mathrm{e}}-1\right) u_{\mathrm{eq}}$ (with $\Gamma_{\mathrm{e}}=4 / 3$ for the relativistic equation of state) are given in Table 2 .

The mean spectra in the strips given in Table 2 were fitted with the three models of the synchrotron radiation and inverse-Compton losses: continuum injection (CI), KardashevPacholczyk (KP), and Jaffe-Perola (JP) (for a review of these models; see Myers \& Spangler (1985)). Assuming that (i) the minimum-energy conditions apply, (ii) the magnetic field $(B)$ in each strip of a lobe is constant throughout the emission process, (iii) the particles are injected into the lobe with a constant power-law energy spectrum with an injection spectral in$\operatorname{dex} \alpha_{\text {inj }}$, and have spent equal time in each strip of different magnetic field strength, the radiative (synchrotron) age in the JP model is given by

$\tau_{\text {rad }}=50.3 \frac{B^{1 / 2}}{B^{2}+B_{\mathrm{iC}}^{2}}\left\{v_{\mathrm{b}}(1+z)\right\}^{-1 / 2}\left[10^{6} \mathrm{yr}\right]$

where $B_{\mathrm{iC}}=0.318(1+z)^{2}=0.479 \mathrm{nT}$ is the magnetic field strength equivalent to the inverse-Compton microwave background, and $v_{\mathrm{b}}$ is the characteristic break frequency corresponding to a break in the particle energy distribution in the emission spectrum. In Eq. (1) $v_{\mathrm{b}}$ is expressed in GHz. It is worth emphasising that the true magnetic field strength $B$ remains one of the most uncertain parameters in the spectral ageing analysis.

Using the SYNAGE software (Murgia 1996), the best fits to the spectra are achieved with the CI model and $\alpha_{\text {inj }}=0.51_{-0.04}^{+0.06}$, and with the KP model and $\alpha_{\text {inj }}=0.53_{-0.02}^{+0.04}$ for the $\mathrm{NE}^{-0.04}$ and SW lobes, respectively. The JP model with $\alpha_{\text {inj }}=0.57_{-0.02}^{+0.04}$ fits quite well the spectrum of the entire source. The above result is consistent with those found in different radio galaxies, for example: the young normal-size Cygnus A (Carilli et al. 1991) and the much older giant 8C 0821+695 (Lara et al. 2000). We find that the reduced $\chi^{2}$ values of the fits with the CI model are lower than those with the KP and JP ones only in the first two strips closer to the NE hotspot. Moreover, different synchrotron ages in the NE lobe resulting from the fits with the above different models, give comparable lobe-material expansion speeds, thus a comparable dynamical age. Note that the fits with the KP and JP models are indistinguishable within the frequency range observed. Thus in the final analysis we use the physically more reasonable JP model fits in both lobes though the KP model formally fits the data slightly better.

The observed spectra in the strips given in Table 2 and their fits with the JP model for $\alpha_{\text {inj }}=0.57$ are shown in Fig. 5. The panels a), b), c) contain the spectra in the NE lobe, and the panels d), e), f) show those in the SW lobe. The values of the resultant break frequency $v_{\mathrm{b}}$ and $\chi_{\text {red }}^{2}$ are shown in each panel. An evident excess of the 8350-MHz flux density value seen in the panels c), d), e) is discussed at the end of Sect. 4.3.

\subsection{Radiative ages}

Following the authors of many previous publications on this topic, in the first step of our analysis we substitute the "equipartition" magnetic field strength $\left(B_{\text {eq }}\right)$ for $B$ in Eq. (1). The resultant spectral ages of particles derived from the data of consecutive strips are given in Col. 4 of Table 3 (indicated as $\tau_{\text {rad }}$ ) and plotted with open circles in Fig. 6 against the distance from the radio core. The errors in $\tau_{\text {rad }}$ are derived from the uncertainties of $B_{\text {eq }}$ and $\nu_{\mathrm{b}}$ given in Tables 2 and 3. The uncertainties in $\alpha_{\text {inj }}$ (given in the text) and $\nu_{\mathrm{b}}$ are estimated from the $1-\sigma$ region of allowance in the free parameters space in the fits. A weighted least-squares fit to the first-step ages in Fig. 6 (shown by the solid lines) yields a mean jet-head separation velocity measured with respect to the lobe emitting plasma, $\left\langle v_{\text {sep }}\right\rangle$, for the $\mathrm{NE}$ and SW lobes. The relevant values divided by the speed of light (indicated as $v_{\text {sep }}^{(1)} / c$ ), and the maximum synchrotron age, $\tau_{\mathrm{rad}}^{\max (1)}$, obtained by extrapolation of these fits up to the core are given in Cols. 5 and 6 of Table 3. These maximum ages are very similar, their mean value is $48.8 \pm 3.9$ Myr. The comparable ages of the lobes would support the presumption that giant radio galaxies are observed at inclination angles very close to $90^{\circ}$. If so, the observed asymmetries in the length, brightness, spectral-index, depolarisation, etc. of the lobes, would likely be caused by an inhomogeneity of the surrounding intergalactic medium, not by the effect of projection and light travel time.

Adopting the formalism used by Lara et al. (2000), we assume that the mean backflow speed $\left(\left\langle v_{\mathrm{bf}}\right\rangle\right)$ is a factor $\epsilon$ of the mean advance velocity of the jet-head with respect to the external medium, i.e. $\left\langle v_{\mathrm{bf}}\right\rangle=\epsilon\left\langle v_{\mathrm{adv}}\right\rangle$. In this case the dynamical age of a source is

$t_{\mathrm{dyn}}=(1+\epsilon) \tau_{\mathrm{rad}}$

Taking into account the results of numerical simulations (e.g. Wilson \& Scheuer 1983) indicating that sources with substantial backflow inflate wider lobes, and the arguments of Alexander \& Leahy (1987) for an increase of the ratio $\left\langle v_{\text {bf }}\right\rangle /\left\langle v_{\text {sep }}\right\rangle$ with decreasing source luminosity, one can assume that the backflow velocity of the emitting plasma down the lobes of $\mathrm{J} 1343+3758$ is comparable to the advance speed of the jet-head because of the low axial ratios of both lobes $(A R<5)$ and their low luminosities. Thus, if $\epsilon=1$ the dynamical age of our giant radio galaxy would be $t_{\mathrm{dyn}}=95 \pm 6 \mathrm{Myr}$.

Under the above assumption, i.e. $\epsilon=1$, the value of $\left\langle v_{\text {adv }}\right\rangle$ would be at least $0.060 \mathrm{c}$. However, if there is a constant 

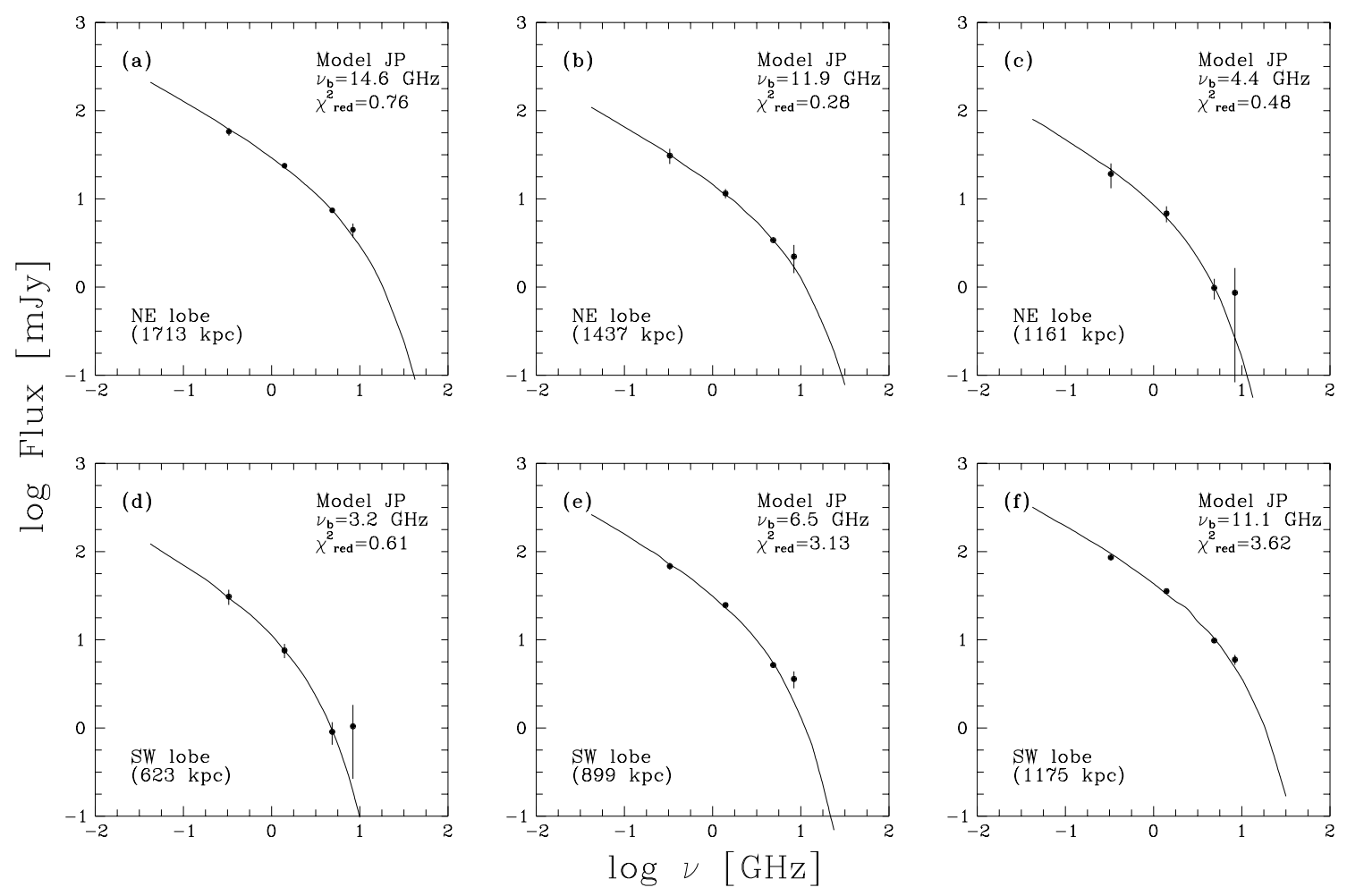

Fig. 5. a)-c) Spectra observed in different strips across the NE lobe of J1343+3758 fitted with the JP model for $\alpha_{\text {inj }}=0.57$. d)-f) The same for the SW lobe. The values of the resultant $\nu_{\mathrm{b}}$ and $\chi_{\text {red }}^{2}$ are shown in the top-right corner of each panel, and the distance of each strip from the radio core is given its bottom-left corner.

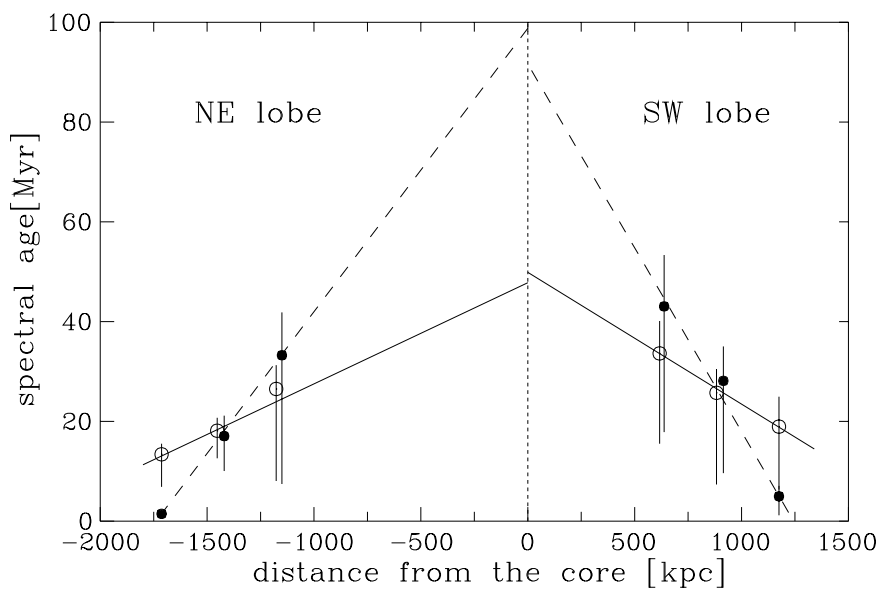

Fig. 6. Radiative age of relativistic particles in the lobes of $\mathrm{J} 1343+3758$ vs. distance from the radio core. Open circles: age corresponding to the equipartition magnetic field strengths; the filled circles: the age determined for the enhanced fields (cf. the text). The solid and dashed lines show the weighted least-squares fits to these ages. The extrapolations of the fits towards the core suggest maximum spectral ages of particles in both lobes.

acceleration or deceleration of the jet-head advance velocity, the mean velocity will be related to the present-day velocity, $v_{\text {adv }}$, as follows:

$v_{\mathrm{adv}}=v_{\mathrm{hi}} \pm a t=2\left\langle v_{\mathrm{adv}}\right\rangle-v_{\mathrm{adv}} \pm a t=\left\langle v_{\mathrm{adv}}\right\rangle \pm \frac{a}{2} t$,

where $v_{\text {hi }}$ is the initial jet-head velocity, and $a$ is the acceleration/deceleration. This is important because an independent estimate of $v_{\text {adv }}$ can be made by assuming the balance between the internal pressure at the jet's head and the ram-pressure of the external ambient medium retreating at speed $v_{\text {adv }}$ (Scheuer 1974). Moreover, basing ourselves on the result obtained by Machalski et al. (2004b), we can assume $v_{\mathrm{adv}} \approx\left\langle v_{\mathrm{adv}}\right\rangle$. In that paper, the authors argue that in the large and old radio sources a jet-head velocity deceleration is negligible because of the evident departure of their lobes/cocoon expansion from selfsimilarity. If so, using the equation (cf. Arnaud et al. 1984)

$\rho_{\mathrm{a}} v_{\mathrm{adv}}^{2}=p_{\min }=u_{\mathrm{eq}} / 3$

it is possible to confine a value of $v_{\text {adv }}$ if the external medium density $\left(\rho_{\mathrm{a}}\right)$ can be estimated.

\subsection{Dependence of the ratio $p_{\min } / \rho_{\mathrm{a}}$ on the dynamical age}

In order to estimate $\rho_{\mathrm{a}}$ in the hotspot regions of $\mathrm{J} 1343+3758$ we used the results of the dynamical evolution analysis for giant radio galaxies of Machalski et al. (2004a). In that paper, the time evolution of giant radio galaxies was analysed on the basis of dynamical evolution of the entire FRII-type population. Using the analytical evolutionary KDA model, the authors derived a number of physical parameters for FRII-type radio sources whose ages had been well determined and published in the literature prior to their analysis.

Summarising, the KDA model assumes that the radio structure is formed by two jets emanating from the AGN into a surrounding ambient medium in two opposite directions, then 
Table 3. Frequency break in the JP model, synchrotron ages and separation speeds derived in the first and the second steps of the ageing analysis.

\begin{tabular}{|c|c|c|c|c|c|c|c|c|c|}
\hline $\begin{array}{c}\text { Distance } \\
{[\mathrm{kpc}]}\end{array}$ & $\begin{array}{c}v_{\mathrm{b}} \\
{[\mathrm{GHz}]}\end{array}$ & $\chi_{\text {red }}^{2}$ & $\begin{array}{c}\tau_{\mathrm{rad}} \\
{[\mathrm{Myr}]}\end{array}$ & $\overline{v_{\mathrm{sep}}^{(1)} / c}$ & $\begin{array}{l}\tau_{\mathrm{rad}}^{\max (1)} \\
{[\mathrm{Myr}]}\end{array}$ & $\begin{array}{c}B_{\text {corr }} \\
{\left[\eta^{-2 / 7} \mathrm{nT}\right]}\end{array}$ & $\begin{array}{c}\tau_{\text {corr }} \\
{[\mathrm{Myr}]}\end{array}$ & $v_{\mathrm{sep}}^{(2)} / c$ & $\begin{array}{l}\tau_{\mathrm{rad}}^{\max (2)} \\
{[\mathrm{Myr}]}\end{array}$ \\
\hline \multicolumn{10}{|l|}{ NE lobe } \\
\hline 1713 & $14.6_{-7.6}^{+10.6}$ & 0.763 & $13.4_{-6.5}^{+2.2}$ & & & $4.00 \pm 0.85$ & $1.5_{-0.9}^{+0.8}$ & & \\
\hline 1437 & $11.9_{-5.2}^{+10.0}$ & 0.280 & $18.1_{-5.5}^{+2.6}$ & & & $0.61 \pm 0.06$ & $17.1_{-7.0}^{+4.1}$ & & \\
\hline \multirow[t]{2}{*}{1161} & $4.4_{-2.4}^{+7.9}$ & 0.480 & $26.5_{-18.5}^{+4.8}$ & & & $0.46 \pm 0.05$ & $33.3_{-25.8}^{+8.6}$ & & \\
\hline & & & & $0.161_{-0.028}^{+0.043}$ & $47.8 \pm 6.6$ & & & $0.057_{-0.001}^{+0.001}$ & $98.8 \pm 1.7$ \\
\hline \multicolumn{10}{|l|}{ SW lobe } \\
\hline 623 & $3.2_{-1.8}^{+4.5}$ & 0.613 & $33.6_{-18.1}^{+6.5}$ & & & $0.34 \pm 0.03$ & $43.0_{-25.2}^{+10.3}$ & & \\
\hline 899 & $6.5_{-3.5}^{+12.0}$ & 3.13 & $25.7_{-18.4}^{+4.8}$ & & & $0.43 \pm 0.04$ & $28.1_{-18.5}^{+6.9}$ & & \\
\hline \multirow[t]{2}{*}{1175} & $11.1_{-6.5}^{+16.0}$ & 3.67 & $18.9_{-12.6}^{+6.0}$ & & & $1.88 \pm 0.22$ & $5.0_{-3.8}^{+2.1}$ & & \\
\hline & & & & $0.123_{-0.051}^{+0.055}$ & $49.9 \pm 1.2$ & & & $0.044_{-0.004}^{+0.005}$ & $91.7 \pm 8.3$ \\
\hline
\end{tabular}
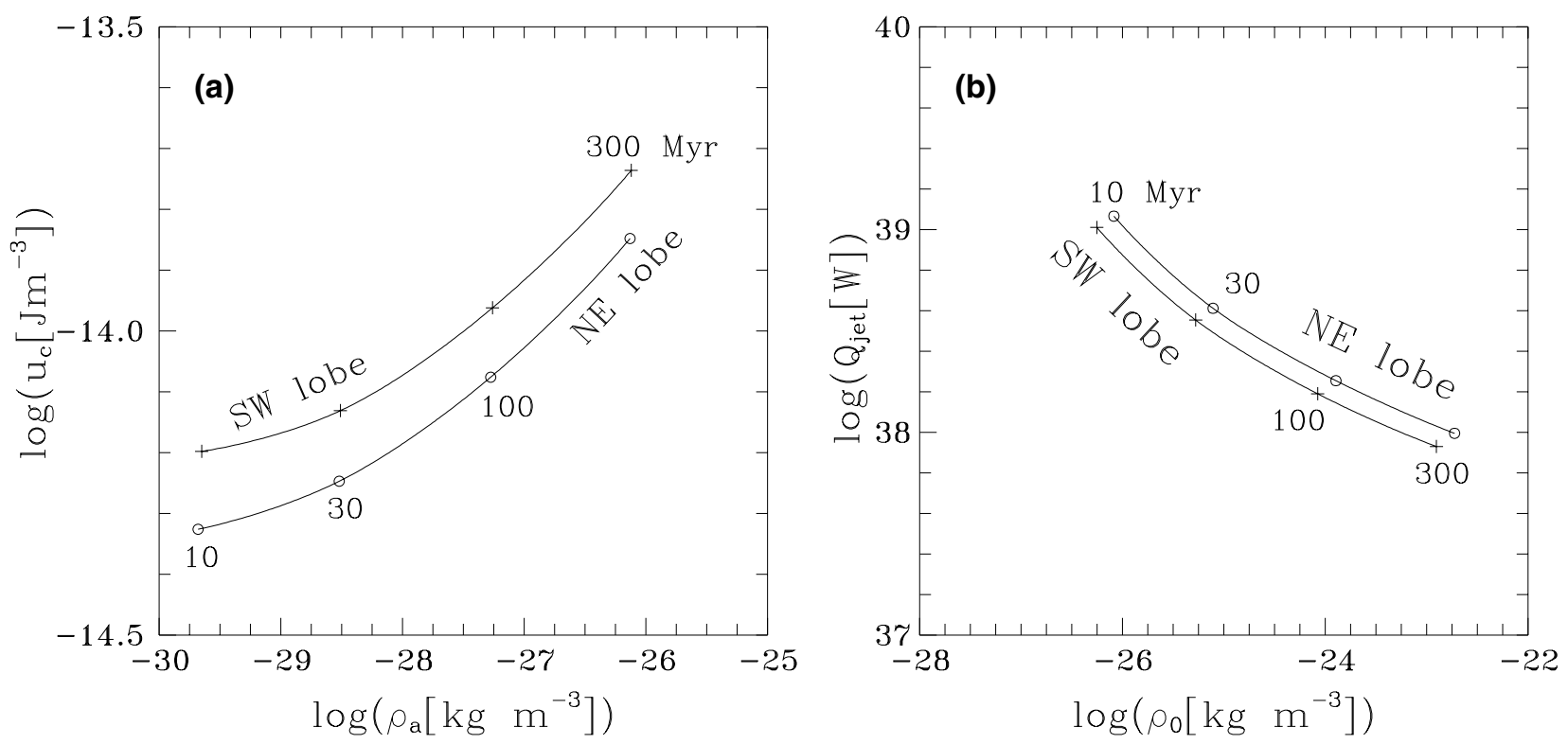

Fig. 7. a) Model energy density $\left(u_{\mathrm{c}}\right)$ in the NE and SW lobes of J1343+3758 vs. the ambient density at their peripheries $\left(\rho_{\mathrm{a}}\right)$ for different ages of the lobes with present values of their observational parameters (cf. the text); b) model jet power $\left(Q_{\text {jet }}\right)$ vs. the central core density $\left(\rho_{0}\right)$ for the NE and SW lobes at different ages.

terminates in strong shocks, and finally inflates the source's cocoon. A density distribution of the unperturbed external gas is approximated by a power-law relation $\rho_{\mathrm{a}}(d)=\rho_{0}\left(d / a_{0}\right)^{-\beta}$, where $d$ is the radial distance from the core, $\rho_{0}$ is the density at the core radius $a_{0}$, and $\beta$ is the exponent in this distribution. The cocoon expands along the jet axis driven by the hotspot pressure $p_{\mathrm{h}}$ and, in the perpendicular direction, by the cocoon pressure $p_{\mathrm{c}}$. The rate at which energy is transported along each jet, $Q_{\text {jet }}$, is assumed to be constant during the source lifetime. The quantitative values of $Q_{\text {jet }}, \rho_{0}$, and the cocoon energy density $u_{\mathrm{c}}$ can be derived by fitting them to the observational data: the monochromatic source luminosity, projected linear size, axial ratio $A R$, and given age ${ }^{2}$.

\footnotetext{
2 The described use of the KDA model differs from that in their original paper where the time evolution of a source parameter (size,
}

Following the approach of Machalski et al. (2004a) and adopting their values of all free parameters of the KDA model (e.g. $a_{0}=10 \mathrm{kpc}$ and $\beta=1.5$ ), we fit the basic physical parameters of the NE and SW lobes of J1343+3758 for a number of dynamical ages. Given these parameters, the relation between $u_{\mathrm{c}}$ and $\rho_{\mathrm{a}}$ is determined at the end of each lobe, and $Q_{\text {jet }}$ and $\rho_{0}$ can be specified. The logarithmic-scale plots of $u_{\mathrm{c}}$ vs. $\rho_{\mathrm{a}}$ and $Q_{\text {jet }}$ vs. $\rho_{0}$ fitted for a number of the lobe ages $t^{*}$, are shown in Figs. $7 \mathrm{a}$ and $7 \mathrm{~b}$, respectively. Note that these plots do not represent a time evolution of the given parameters; they show their predicted values at different (unknown) age of the lobes with apparent size, axial ratio, and luminosity.

luminosity, energy, pressure, etc.) can be predicted by the model with all its free parameters fixed. 
Inserting the values of $u_{\mathrm{c}}$ into Eq. (3) instead of $u_{\mathrm{eq}}$ (which may be valid for the unknown present age of the lobe only), we have

$v_{\mathrm{adv}}\left(t^{*}\right)=\left(\frac{u_{\mathrm{c}}\left(t^{*}\right)}{3 \rho_{\mathrm{a}}\left(t^{*}\right)}\right)^{1 / 2}$.

As both $u_{\mathrm{c}}$ and $\rho_{\mathrm{a}}$ appear to be power-law functions of $t^{*}$, we find the following dependence of $v_{\mathrm{adv}} / c$ on age $t$ (in Myr):

$\log \left(v_{\mathrm{adv}} / c\right)=(-0.9982 \pm 0.0007) \log t^{*}-(0.1161 \pm 0.0013)$,

$\log \left(v_{\mathrm{adv}} / c\right)=(-0.9987 \pm 0.0014) \log t^{*}-(0.0651 \pm 0.0027)$

for the NE and SW lobes, respectively. Inserting the first step values of $\tau_{\mathrm{rad}}^{\max (1)}$ (Col. 6 in Table 3 ) into the above equations, we find advance speeds of $(0.016 \pm 0.003) c$ and $(0.017 \pm 0.005) c$ for the NE and SW lobes, respectively.

If these were correct, the backflow velocity $\left\langle v_{\text {bf }}\right\rangle$ would be a few times higher than the $\left\langle v_{\text {adv }}\right\rangle$ speeds, i.e. $\epsilon>1$. This problem already occurred in the spectral ageing analysis of Cygnus A by Carilli et al. (1991). In their paper they proposed a "selfconsistent" solution allowing a decrease of the magnetic field strengths which would increase the jet-head pressure and slow the rate of radiative losses, and hence would decrease the separation speed of the lobe material.

A similar solution for the case of $\mathrm{J} 1343+3758$ leads to a different conclusion. Though either decrease or increase of the magnetic field strength with respect to the equipartition value would raise both the total pressure of the relativistic particles and of the $B$-fields according to the relation $U_{\text {tot }}=$ $(4 / 3) E_{\mathrm{e}}+E_{\mathrm{m}} \propto(4 / 3) B^{-3 / 2}+B^{2}$, a reduction of the lobematerial separation speed, in order to match the different velocities $\left\langle v_{\text {sep }}\right\rangle$ and $\left\langle v_{\text {adv }}\right\rangle$, is only possible by an increase of the magnetic field strength. This is a consequence of Eq. (1): the maximum amount of time elapsed since the last acceleration of particles is achieved for $B^{*}=B_{\mathrm{iC}} / \sqrt{3}$ and is a function of redshift. If the equipartition fields $\left(B_{\text {eq }}\right)$ are lower than the value of $B^{*}$, their increase raises $\tau_{\text {rad }}$; if $B_{\text {eq }}$ is higher than $B^{*}$, its further increase reduces $\tau_{\text {rad }}$. Low equipartition magnetic fields are typical of almost all giant radio sources because of their large volumes and low luminosities.

Therefore in the second step of our analysis, we increase the fields $B_{\text {eq }}$ and find that a satisfactory match between $\left\langle v_{\text {sep }}\right\rangle$ and $\left\langle v_{\text {adv }}\right\rangle$ would be achieved if the fields were enhanced by a factor of 5.5 and 3.4 in the NE and SW lobes, respectively. These enhanced values (indicated $B_{\text {corr }}$ ) are given in Col. 7 of Table 3 . The relevant values of the radiative age of relativistic particles $\left(\tau_{\text {corr }}\right)$ vs. the distance from the radio core are given in Col. 8 of Table 3, and plotted with filled circles in Fig. 6. The weighted least-squares fit to the second-step ages (shown by the dashed lines in Fig. 6) yields much slower velocities $\left\langle v_{\text {sep }}\right\rangle$ than the corresponding values found in the first step. These values in units of $c,\left(v_{\mathrm{sep}}^{(2)} / c\right)$, and the maximum radiative age at the core position are given in Cols. 9 and 10 of Table 3.

Now, multiplying the right-hand side of Eq. (3) by the factors of 13.01 and 5.05 (as a result of the $B_{\text {eq }}$-field enhancement by 5.5 and 3.4$)$, the previous $\log \left(v_{\text {adv }} / c\right)-t^{*}$ relations change into:

$\log \left(v_{\mathrm{adv}} / c\right)=(-0.9982 \pm 0.0007) \log t^{*}+(0.4411 \pm 0.0013)$, $\log \left(v_{\mathrm{adv}} / c\right)=(-0.9987 \pm 0.0014) \log t^{*}+(0.2867 \pm 0.0027)$

for the NE and SW lobes, respectively. Inserting the maximum values of particle ages determined in the second step (Col. 10 of Table 3 ) into the above relations, we have $v_{\mathrm{adv}}=(0.028 \pm 0.007) c$ and $v_{\mathrm{adv}}=(0.021 \pm 0.013) c$ for the NE and SW lobes, respectively.

Indeed, these speeds are one half of the second step separation velocities (see Col. 9 of Table 3) as required by the assumption of comparable jet-head and backflow velocities. Dividing the projected lobe lengths of $1800 \pm 18 \mathrm{kpc}$ and $1340 \pm 18 \mathrm{kpc}$ by the averaged advance velocities of $(0.0285 \pm$ $0.0033) c$ and $(0.0217 \pm 0.0066) c$, and dynamical ages of $206 \pm 22$ and $201 \pm 58 \mathrm{Myr}$, respectively, for the NE and SW lobes are found. As they are very close to each other, the mean value of $204 \pm 40 \mathrm{Myr}$ seems to be a reliable dynamical age of the radio galaxy $\mathrm{J} 1343+3758$. If correct, its spectral age of about 49 Myr will be only one fourth of the suggested dynamical age.

The spectra in Fig. 5 show an intriguing excess of the $8350 \mathrm{MHz}$ flux-density in faint parts of the extended diffuse structure of both lobes. It could be explained by a slight but possible deficit of $4860 \mathrm{MHz}$ flux density due to missing spacings in the high-frequency VLA observations. We estimate a possible decrease of that flux at the distance from the core of 1161 and $623 \mathrm{kpc}$ in the NE and SW lobes, respectively, to be less than $20 \%$. This would cause about $50 \%$ higher values of $v_{\mathrm{b}}$ and about $22 \%$ lower values of $\tau_{\text {rad }}$ and $\tau_{\text {corr }}$ at these distances, resulting in less than $10 \%$ decrease of the $\tau_{\mathrm{rad}}^{\max (1)}$ and $\tau_{\mathrm{rad}}^{\max (2)}$ values given in Table 3. However, similar high-frequency excesses seem to be present in some other giant radio galaxies, e.g. in the southern lobe of B1358+305 (Parma et al. 1996), in both lobes of $8 \mathrm{C} 0821+695$ (Lara et al. 2000), and possibly in one of the lobes of the giant quasars MCR 0437-244 and MCR 1025-229 (Ishwara-Chandra \& Saikia 1999). More high-frequency and adequate angular-resolution data are necessary to determine whether such high-frequency flattening could be real.

\subsection{Further dynamical consideration}

A multiplication of the left-hand side of Eq. (3) by the jethead area yields the ram-pressure force exerted by the ambient medium and balancing the jet thrust. Begelman \& Cioffi (1989) modified the former Scheuer model of an overpressured cocoon (Model A; Scheuer 1974), admitting fluctuations in the jet direction that cause the time-averaged momentum flux to be spread over a much wider area $A_{\mathrm{h}}$ than the instantaneous cross-section of the jet. That modified model makes it possible to predict the time elapsed from the 'birth' of the source to the epoch of our observations when it reached the observed linear size. This time, i.e. dynamical age (their Eq. (3)), is

$t_{\text {dyn }} \approx A_{\mathrm{c}}\left(\frac{\rho_{\mathrm{a}}}{Q_{\text {jet }} v_{\text {jet }} A_{\mathrm{h}}}\right)^{1 / 4}$

where $v_{\text {jet }}$ is the jet speed and $A_{\mathrm{h}}$ and $A_{\mathrm{c}}$ are the cross sectional areas of the bow shock at the end of the lobe and of the lobe itself measured transversely to the jet axis, respectively. In most analyses $v_{\text {jet }}$ is assumed equal to the speed of light. 
$A_{\mathrm{h}}$ and $A_{\mathrm{c}}$ can be determined from the observations. For an independent estimation of the dynamical age from Eq. (4), the expected values of $Q_{\text {jet }}$ and $\rho_{\mathrm{a}}$ are taken from the statistical correlations found in the sample of FRII-type radio sources by Machalski et al. (2004a) (cf. Sect. 4.3). They found that $Q_{\text {jet }}$ is highly correlated with the source luminosity $L_{1.4}$, and $\rho_{\mathrm{a}}$ is highly anticorrelated with its linear size $D$. The partial correlation $Q_{\text {jet }}-L_{1.4}$ was calculated with taking account of unavoidable correlations of these two parameters with redshift and source age. A partial correlation coefficient of +0.901 was found if $z$ and $t_{\mathrm{dyn}}$ were kept constant. A partial correlation coefficient of -0.935 in the correlation $\rho_{\mathrm{a}}-D$ was found if $\rho_{0}$ and $t_{\text {dyn }}$ were kept constant. Given $L_{1.4}$ and $D$, the values of $Q_{\text {jet }}$ and $\rho_{\mathrm{a}}$ for the entire giant source can be estimated from:

$\log \left(Q_{\mathrm{jet}}[\mathrm{W}]\right)=0.768 \log \left(L_{1.4}\left[\mathrm{~W} \mathrm{~Hz}^{-1} \mathrm{sr}^{-1}\right]\right)+19.08$ and $\log \left(\rho_{\mathrm{a}}\left[\mathrm{kg} \mathrm{m}^{-3}\right]\right)=-1.5 \log (D[\mathrm{Mpc}])-25.82$.

For J1343+3758 we have: $L_{1.4}=2.63 \times 10^{24} \mathrm{~W} \mathrm{~Hz}^{-1} \mathrm{sr}^{-1}$ and $D=3.14 \mathrm{Mpc}$, hence $Q_{\text {jet }}=6.83 \times 10^{37} \mathrm{~W}$ and $\rho_{\mathrm{a}}=2.72 \times$ $10^{-27} \mathrm{~kg} \mathrm{~m}^{-3}$ are found from the above statistical correlations. The largest lateral deconvolved size of the source measured in the maps is $506 \pm 86 \mathrm{kpc}$, which yields $A_{\mathrm{c}}=(1.88 \pm 0.32) \times$ $10^{44} \mathrm{~m}^{2}$. The hotspot in the NE lobe has the minor Gaussian diameter of 2'.7 $70^{\prime} \cdot 1$ measured in three maps (cf. Sect. 3.2) yielding $A_{\mathrm{h}}=(1.15 \pm 0.04) \times 10^{41} \mathrm{~m}^{2}$. Substitution of the above values into Eq. (4) with approximation $v_{\text {jet }} \approx c$ gives $t_{\text {dyn }} \approx$ $195 \mathrm{Myr}$, fully consistent with the value estimated in Sect. 4.3.

\section{Other physical parameters}

The main physical parameters for both individual lobes of $\mathrm{J} 1343+3758$ derived from fitting are given in Table 4, and have been used to compare with the relevant quantities obtained from the statistics given in Sect. 4.4. The values of $Q_{\text {jet }}, \rho_{0}$ and $\rho_{\mathrm{a}}$, as well as the mean energy density $\left(u_{\mathrm{c}}\right)$, total emitted energy $\left(E_{\mathrm{t}}-\right.$ the product of energy density and the lobe/cocoon volume), and the ratio of the total energy delivered by the jet and the emitted energy $\left(Q_{\text {jet }} t_{\mathrm{dyn}} / E_{\mathrm{t}}\right)$ derived for the NE and SW lobes at ages of 206 and $201 \mathrm{Myr}$, respectively, are given in Table 4. The latter ratio, i.e. the reciprocal of the efficiency factor by which the kinetic energy of the jets is converted into electromagnetic radiation, is much higher than $\sim 2$, a value usually assumed. The difference between the values of $Q_{\text {jet }}(\sim 20 \%)$ and $\rho_{0}(\sim 60 \%)$ found from fitting for the opposite lobes can be considered as the uncertainties of these values derived from the model.

Comparing the values of $Q_{\text {jet }}$ and $\rho_{\mathrm{a}}$ from Table 4 to the corresponding values derived from the statistical correlations in Sect. 4.4, one can notice that the jet power and the ambient density required by the dynamical model for the lobes of $\mathrm{J} 1343+3758$ are higher by $\sim 60 \%$ and $\sim 10 \%$, respectively, than the values suggested by the statistics. However these differences have a marginal influence on the source dynamical age derived from Eq. (4) where it is proportional to $\left(\rho_{\mathrm{a}} / Q_{\mathrm{jet}}\right)^{1 / 4}$. Now, the substitution of the $Q_{\text {jet }}$ and $\rho_{\mathrm{a}}$ values fitted for the NE lobe (containing the distinct hotspot) into Eq. (4) gives
Table 4. Main physical parameters of the lobes derived from the analytical model of KDA (cf. Sect. 4.3) assuming the dynamical age estimate determined in this paper.

\begin{tabular}{|c|c|c|c|}
\hline Parameter & "Units & $\begin{array}{c}\text { NE lobe } \\
t=206 \mathrm{Myr}\end{array}$ & $\begin{array}{c}\text { SW lobe } \\
t=201 \mathrm{Myr}\end{array}$ \\
\hline Jet power & $Q_{\text {jet }}[\mathrm{W}]$ & $9.73 \times 10^{37}$ & $1.20 \times 10^{38}$ \\
\hline Central core density & $\rho_{0}\left[\mathrm{~kg} \mathrm{~m}^{-3}\right]$ & $7.34 \times 10^{-24}$ & $4.55 \times 10^{-24}$ \\
\hline ambient density & $\rho_{\mathrm{a}}\left[\mathrm{kg} \mathrm{m}^{-3}\right]$ & $3.02 \times 10^{-27}$ & $2.90 \times 10^{-27}$ \\
\hline Lobe energy density & $u_{\mathrm{c}}\left[\mathrm{J} \mathrm{m}^{-3}\right]$ & $1.15 \times 10^{-14}$ & $1.45 \times 10^{-14}$ \\
\hline Lobe emitted energy & $E_{\mathrm{t}}[\mathrm{J}]$ & $6.76 \times 10^{52}$ & $9.64 \times 10^{52}$ \\
\hline $\begin{array}{l}\text { Ratio of delivered } \\
\text { to emitted energy }\end{array}$ & $Q_{\text {jet }} t / E_{\mathrm{t}}$ & $9.4 \pm 2.6$ & $7.9 \pm 2.2$ \\
\hline
\end{tabular}

$t_{\mathrm{dyn}}=183 \mathrm{Myr}$, sufficiently close to the estimate determined from the "self-consistent" solution in Sect. 4.3.

\section{Summary}

In this paper the radio properties of the giant radio galaxy $\mathrm{J} 1343+3758$ have been analysed. We present the new high frequency 4860 and $8350 \mathrm{MHz}$ total power and polarisation maps of this source. Using the flux densities between 151 and $8350 \mathrm{MHz}$, and radio maps at 327, 1400, 4860 and $8350 \mathrm{MHz}$ we have studied the synchrotron spectrum in different parts of its extended lobes, and performed the standard ageing analysis. The maximum age of the relativistic particles calculated with the assumption of equipartition magnetic fields is found to be about $50 \mathrm{Myr}$ which is only one fourth of the estimated dynamical age of the radio structure derived from the ram-pressure arguments. This result is in agreement with the conclusion of Blundell \& Rawlings (2000) that the spectral and dynamical ages of a FRII-type radio galaxy are comparable only when these ages are $\ll 10 \mathrm{Myr}$ and the discrepancy between them increases with the age of the radio source. We include here also the KDA model estimates of other physical parameters of $\mathrm{J} 1343+3758$, i.e. the jet power, the central density of the galaxy nucleus, the ambient medium density, the lobe energy density and the total energy of the source.

Acknowledgements. The authors acknowledge the National Radio Astronomy Observatory (NRAO) which is a facility of the National Science Foundation operated under cooperative agreement by Associated Universities, Inc. as well as the Space Telescope Science Institute for the usage of the DSS data base. This research is partly based on observations performed with the 100-m telescope of the Max-Planck-Institut für Radioastronomie (MPIfR) at Effelsberg. M.J. acknowledges the Deutsche Forschungsgemeinschaft for the award of a postdoctoral fellowship (GRK 787).

\section{References}

Alexander, P., \& Leahy, J. P. 1987, MNRAS, 225, 1

Arnaud, K. A., Fabian, A. C., Eales, S. A., Jones, C., \& Forman, W. 1984, MNRAS, 211, 981

Baars, J. W. M., Genzel, R., Pauliny-Toth, I. I. K., \& Witzel, A. 1977, A\&A, 61, 99

Becker, R. H., White, R. L., \& Helfand, D. J. 1995, ApJ, 450, 559 (FIRST) 
Begelman, M. C., \& Cioffi, D. F. 1989, ApJ, 345, L21

Blundell, K. M., Rawlings, S., \& Willott, C. J. 1999a, AJ, 117, 766

Blundell, K. M., \& Rawlings, S. 1999b, Nature, 399, 330

Blundell, K. M., \& Rawlings, S. 2000, AJ, 119, 1111

Carilli, C. L., Perley, R. A., Dreher, J. W., \& Leahy, J. P. 1991, ApJ, 383,554

Condon, J. J., Cotton, W. D., Greisen, E. W., et al. 1998, AJ, 115, 1693 (NVSS)

Cotter, G. 1998, in Observational Cosmology with the New Radio Surveys, ed. M. N. Bremer, N. Jackson, \& I. Perez-Fournon (Kluwer Acad. Publ.), 233

Fanaroff, B. L., \& Riley, J. M. 1974, MNRAS, 167, 31

Gregory, P. C., Scott, W. K., Douglas, K., \& Condon, J. J. 1996, ApJS, 103, 427 (GB6)

Hales, S. E. G., Baldwin, J. E., \& Warner, P. J. 1988, MNRAS, 234, 919 (6C)

Haslam, C. G. T. 1974, A\&AS, 15, 333

Ishwara-Chandra, C. H., \& Saikia, D. J. 1999, MNRAS, 309, 100

Jones, T. W., Ryu, D., \& Engel, A. 1999, ApJ, 512, 105

Jaffe, W. J., \& Perola, G. C. 1973, A\&A, 26, 423

Kaiser, C. R., Dennett-Thorpe, J., \& Alexander, P. 1997, MNRAS, 292, 723 (KDA)

Kaiser, C. R. 2000, A\&A, 362, 447

Kardashev, N. S. 1962, SvA, 6, 317

Klein, U., Mack, K.-H., Gregorini, L., \& Parma, P. 1995, A\&A, 303, 427

Lara, L., Mack, K.-H., Lacy, M., et al. 2000, A\&A, 356, 63

Leahy, J. P., \& Williams, A. G. 1984, MNRAS, 210, 929

Leahy, J. P., Muxlow, T. W. B., \& Stephens, P. W. 1989, MNRAS, 239, 401

Liu, R., Pooley, G., \& Riley, J. M. 1992, MNRAS, 257, 545

Longair, M. S., \& Riley, J. M. 1979, MNRAS, 188, 625
Machalski, J., \& Jamrozy, M. 2000, A\&A, 363, L17

Machalski, J., Jamrozy, M., \& Zola, S. 2001, A\&A, 371, 445

Machalski, J., Chyży, K. T., \& Jamrozy, M. 2004a, Acta Astr., 54, 249

Machalski, J., Chyży, K. T., \& Jamrozy, M. 2004b, Acta Astr., 54, 391

Mack, K.-H., Klein, U., O'Dea, C. P., Willis, A. G., \& Saripalli, L. 1998, A\&A, 329, 431

Miley, G. 1980, ARA\&A, 18, 165

Murgia, M. 1996, Laurea Thesis, University of Bologna

Myers, S. T., \& Spangler, S. R. 1985, ApJ, 291, 52

Neeser, M. J., Eales, S. A., Law-Green, J. D., Leahy, J. P., \& Rawlings, S. 1995, ApJ, 451, 76

Pacholczyk, A. G. 1970, Radio Astrophysics (San Francisco: Freeman)

Parma, P., de Ruiter, H. R., Mack, K.-H., et al. 1996, A\&A, 311, 49

Parma, P., Murgia, M., Morganti, R., et al. 1999, A\&A, 344, 7

Pooley, D. M., Waldram, E. M., \& Riley, J. M. 1998, MNRAS, 298, 637

Rengelink, R. B., Tang, Y., de Bruyn, A. G., et al. 1997, A\&AS, 124, 259 (WENSS)

Rudnick, L., Katz-Stone, D. M., \& Anderson, M. C. 1994, ApJS, 90, 955

Saikia, D. J., \& Salter, C. J. 1988, ARA\&A, 26, 93

Scheuer, P. A. G. 1974, MNRAS, 166, 513

Schoenmakers, A. P., Mack, K.-H., Lara, L., et al. 1998, A\&A, 336, 455

Schoenmakers, A. P., Mack, K.-H., de Bruyn, A. G., et al. 2000, A\&AS, 146, 293

Sieber, W., Haslam, C. G. T., \& Salter, C. J. 1979, A\&A, 74, 361

Simard-Normandin, M., \& Kronberg, P. P. 1980, ApJ, 242, 74

Wilson, M. J., \& Scheuer, P. A. G. 1983, MNRAS, 205, 449

Zhang, X., Zheng, Y., Chen, H., et al. 1997, A\&AS, 121, 59 